\title{
The current understanding of immunotoxicity induced by silica nanoparticles
}

\author{
Chen Liangjiao', Kang Yiyuan², Guan Hongbing ${ }^{1}$, Liu Jia², Zhong Wenchao', Zhang Yanli² \\ \& Shao Longquan $*, 2$ \\ ${ }^{1}$ Key Laboratory of Oral Medicine, Guangzhou Institute of Oral Disease, Stomatology Hospital of Guangzhou Medical University, \\ Guangzhou 510140, PR China \\ ${ }^{2}$ Stomatology department, Nanfang Hospital, Southern Medical University, Guangzhou 510515, PR China \\ *Author for correspondence: Tel.: 86-20-62787153; Fax: 86-20-61641101; shaolongquan@smu.edu.cn
}

First draft submitted: 11 March 2019; Accepted for publication: 21 March 2019; Published online: 14 May 2019

Keywords: adjuvants $\bullet$ allergic responses $\bullet$ autophagy $\bullet$ cytotoxicity $\bullet$ immune system $\bullet$ immunotoxicity $\bullet$ inflammatory cells $\bullet$ phagocytes $\bullet$ ROS production $\bullet$ silica nanoparticles

Silica nanoparticles (SiNPs) are one of the three most produced nanoparticles and are widely used in dietary supplements, vaccine adjuvants, dental fillers and drug delivery. However, the toxicity of SiNPs to the immune system has received an increasing amount of attention. SiNPs may have toxic effects on phagocytes, particularly macrophages, dendritic cells (DCs) and T-lymphocytes. Additionally, the immunotoxicity of SiNPs to tissues and organs has been investigated in vivo. The safety and potential adverse effects of SiNPs on the immune system have been partially reviewed in our previous paper [1]. As new research has developed, we have a new understanding of the immunotoxicity of SiNPs.

\section{Recent advances in immunotoxicity studies}

The cytotoxicity of SiNPs to the immune system is determined in part by the physicochemical properties of the particles. For example, SiNPs have exhibited dose- and time-dependent toxicity to human lymphocytes in vitro [2,3]. Gene expression analysis results have shown that mesoporous SiNPs are capable of changing gene expression in RAW264.7 cells at nontoxic doses, while a minimum effect on gene transcription is observed for nonporous SiNPs [4]. Additionally, $10 \mathrm{~nm}$ SiNPs could induce hepatotoxicity by hyperplasia of Kupffer cells and infiltration of inflammatory cells in vivo [5]. Research on the direct influence of SiNPs on bone marrow, the thymus or lymph nodes, which are immune organs, is rare.

In addition to the toxicity of the particles themselves, their combined toxicity with other factors should also be considered. It has been reported that co-treatment with SiNPs and benzoapyrene $(\mathrm{B}[\mathrm{a}] \mathrm{P})$ has a more severe toxic effect on the immune system than their individual exposures in zebrafish embryos. The signalling pathways associated with this toxicity are the MAPK pathway, p 53 pathway, PI3k/Akt signalling pathway and several pathways related to the immune response [6]. In addition, the presence of both SiNPs and bacteria may increase immune toxicity. Extracellular media (EMs) containing bacteria and SiNPs synergistically increased cytotoxicity toward and cytokine production of dendritic cells (DCs), which was influenced by the nanoparticle dose and bacterial species. In the presence of Escherichia coli and Staphylococcus epidermidis, nontoxic doses of SiNPs promoted the secretion of pro-inflammatory mediators, such as IL-1 $\beta$, IL-12 and IL-6, while toxic doses of SiNPs stimulated the secretion of IFN- $\gamma$, TNF- $\alpha$, IL-4 and IL-5 [7]. Extracellular media containing Pseudomonas aeruginosa and Staphylococcus aureus, which are pathogenic bacteria, induced the production of cytokines only when they were simultaneously mixed with toxic concentrations of SiNPs [7]. Furthermore, the presence of SiNPs increases the susceptibility to bacterial infection. Exposure of lung tissue to SiNPs and silicosis tended to increase tuberculosis infections and their progression to active tuberculosis [8].

However, the exact mechanisms of these effects are not yet fully understood. In addition to the pro-inflammatory effect, ROS production and autophagy changes have been considered the main mechanisms of immunotoxicity [1].

Future 8 Medicine 
High expression in immune cells of transient receptor potential (TRP) ion channels, which are a superfamily of cation channels with many biofunctions, is one of the underlying mechanisms of toxicity. TRPM2 and TRPM8 channel expression changed significantly after exposure to small mesoporous SiNPs, while mesoporous SiNPs have shown TRPA1-related toxicity [9].

\section{Future prospects \& limitations}

Although SiNPs have intrinsic toxicity, which often limits their clinical use, there are advantages to this toxic effect. Cationic silica nanoparticles (CSiNPs) can destroy tumors in vivo and stimulate the immune response when combined with molecular adjuvants, which activates interferon gene stimulators in antigen-presenting cells [10]. Recently, a study was focused on the modulating effects of SiNPs on the immune response, which may activate or inhibit immunity. Negatively and neutrally charged SiNPs $(<200 \mathrm{~nm})$ showed an immunosuppressive effect, while positively charged SiNPs did not influence the immune response [11]. This effect may mitigate allergic responses in skin. It is well known that SiNPs, which are 10-20 nm in diameter, can activate mononuclear phagocytes. Furthermore, SiNPs that are $<10 \mathrm{~nm}$ in diameter induced dose-dependent CD 4 and CD8 T-cell activation [12].

Using the immunomodulatory effect of SiNPs, mesoporous SiNPs doped with $\mathrm{Ca}, \mathrm{Mg}$ and $\mathrm{Zn}$ promoted the anticancer immune response and increased $\mathrm{CD}^{+}$and $\mathrm{CD}^{+}$T-cell populations in the spleen [13]. Amorphous SiNPs (with a diameter of $33 \mathrm{~nm}$ ) had an adjuvant effect on the responses of T helper cell, such as Th1, Th2 and Th17 [14]. It has been reported that asymmetric mesoporous SiNPs with a head-tail structure, compared with those that have a symmetric structure, have a greater potential for delivering peptide antigens, stimulating a stronger antibody immune response [15]. Understanding the effects of SiNPs on immune response and adjuvanticity might guide the exploitation of novel vaccine adjuvants and therapies for allergic diseases.

From what has been discussed above, in addition to focusing on the toxicity of the particles themselves, it is also necessary to consider their synergistic effects with other toxins and bacteria in the environment. The direct influence of SiNPs on immune system organs and their genotoxicity to immune cells should be further studied. The mechanism of their immunotoxicity, which is caused by a complex set of factors, should also be further researched. In certain situations, the effects and toxicity of nanoparticles on the immune system may be used as an advantage. However, when SiNPs are used as an antitumoral adjuvant, their effects on normal cells should also be investigated. The purity of SiNPs should be evaluated carefully when used as vaccine adjuvants. Contamination of SiNPs from surfactants, such as cetyltrimethyl ammonium bromide (CTAB) or cetyltrimethylammoniumchloride (CTAC), which are used in the synthesis of mesoporous silica, may cause serious damage to tissues.

\section{Financial \& competing interests disclosure}

This work was supported by the National Natural Science Foundation of China (81600904), the fund of Guangzhou Science Technology and innovation commission (201707010026) and the fund of Guangzhou Medical University (B185004177). The authors have no other relevant affiliations or financial involvement with any organization or entity with a financial interest in or financial conflict with the subject matter or materials discussed in the manuscript apart from those disclosed.

No writing assistance was utilized in the production of this manuscript.

\section{References}

Papers of special note have been highlighted as: $\bullet$ of interest; $\bullet \bullet$ of considerable interest.

1. Chen LJ, Liu J, Zhang YL et al. The toxicity of silica NPs to the immune system. Nanomedicine 13(15),1939-1962 (2018).

- The potential adverse effects of silica nanoparticles (SiNPs) on the immune system have been partially reviewed in this paper.

2. Azimipour S, Ghaedi S, Mehrabi Z et al. Heme degradation and iron release of hemoglobin and oxidative stress of lymphocyte cells in the presence of silica nanoparticles. Int. J. Biol. Macromol. 118(Pt A), 800-807 (2018).

3. Sabziparvar N, Saeedi $\mathrm{Y}$, Nouri M et al. Investigating the interaction of silicon dioxide nanoparticles with human hemoglobin and lymphocyte cells by biophysical, computational, and cellular studies. J. Phys. Chem. B 122(15), 4278-4288 (2018).

4. Yazdimamaghani M, Moos PJ, Ghandehari H. Global gene expression analysis of macrophage response induced by nonporous and porous silica nanoparticles. Nanomedicine 14(2), 533-545 (2018).

5. Almansour M, Alarifi S, Jarrar B. In vivo investigation on the chronic hepatotoxicity induced by intraperitoneal administration of 10-nm silicon dioxide nanoparticles. Int. J. Nanomed. 13, 2685-2696 (2018).

6. Asweto CO, Hu H, Liang S et al. Gene profiles to characterize the combined toxicity induced by low level co-exposure of silica nanoparticles and benzo[a]pyrene using whole genome microarrays in zebrafish embryos. Ecotoxicol. Environ. Saf. 163, 47-55 (2018).

-. In addition to the toxicity of the particles themselves, the combined toxicity with benzo[a] pyrene should also be considered. 
7. Malachin G, Lubian E, Mancin F, Papini E, Tavano R. Combined action of human commensal bacteria and amorphous silica nanoparticles on the viability and immune responses of dendritic cells. Clin. Vaccine Immunol. 4(10), pii: e00178-17 (2017).

-. The combined action of human commensal bacteria and amorphous SiNPs to immune cells should also be considered.

8. Konečný P, Ehrlich R, Gulumian M, Jacobs M. Immunity to the dual threat of silica exposure and mycobacterium tuberculosis. Front. Immunol. (2019). https://www.ncbi.nlm.nih.gov/pmc/articles/PMC6334662/

- Exposure of lung tissue to SiNPs and silicosis tended to increase tuberculosis infections and their progression to active tuberculosis.

9. Mohammadpour R, Yazdimamaghani M, Reilly C, Ghandehari H. Transient receptor potential (TRP) ion channel-dependent toxicity of silica nanoparticles and Poly(amido amine) (PAMAM) dendrimers. J. Pharmacol. Exp. Ther. (2018). http://jpet.aspetjournals.org/content/early/2019/05/20/jpet.119.257105

- $\quad$ TRPM2 and TRPM8 channel expression changed significantly after exposure to small mesoporous SiNPs.

10. An M, Yu C, Xi J et al. Induction of necrotic cell death and activation of STING in the tumor microenvironment via cationic silica nanoparticles leading to enhanced antitumor immunity. Nanoscale 10(19), 9311-9319 (2018).

- SiNPs leading to enhanced antitumor immunity.

11. Jatana S, Palmer BC, Phelan SJ, DeLouise LA. Immunomodulatory: effects of nanoparticles on skin allergy. Sci. Rep. 7(1), 3979 (2017).

12. Vis B, Hewitt RE, Faria $\mathrm{N}$ et al. Non-functionalized ultrasmall silica nanoparticles directly and size-selectively activate T cells. ACS Nano 12(11), 10843-10854 (2018).

- SiNPs directly and size-selectively activate T cells.

13. Wang X, Li X, Ito A et al. Biodegradable metal ion-doped mesoporous silica nanospheres stimulate anticancer Th1 immune response in vivo. ACS Appl. Mater. Interfaces 9(50), 43538-43544 (2017).

14. Toda T, Yoshino S. Enhancement of ovalbumin-specific Th1, Th2, and Th17 immune responses by amorphous silica nanoparticles. Int J. Immunopathol. Pharmacol. 29(3), 408-420 (2016).

15. Abbaraju PL, Jambhrunkar M, Yang Y, Liu Y, Lu Y, Yu C. Asymmetric mesoporous silica nanoparticles as potent and safe immunoadjuvants provoke high immune responses. Chem. Commun. 54(16), 2020-2023 (2018).

- Asymmetric mesoporous SiNPs provoke high immune responses. 
